
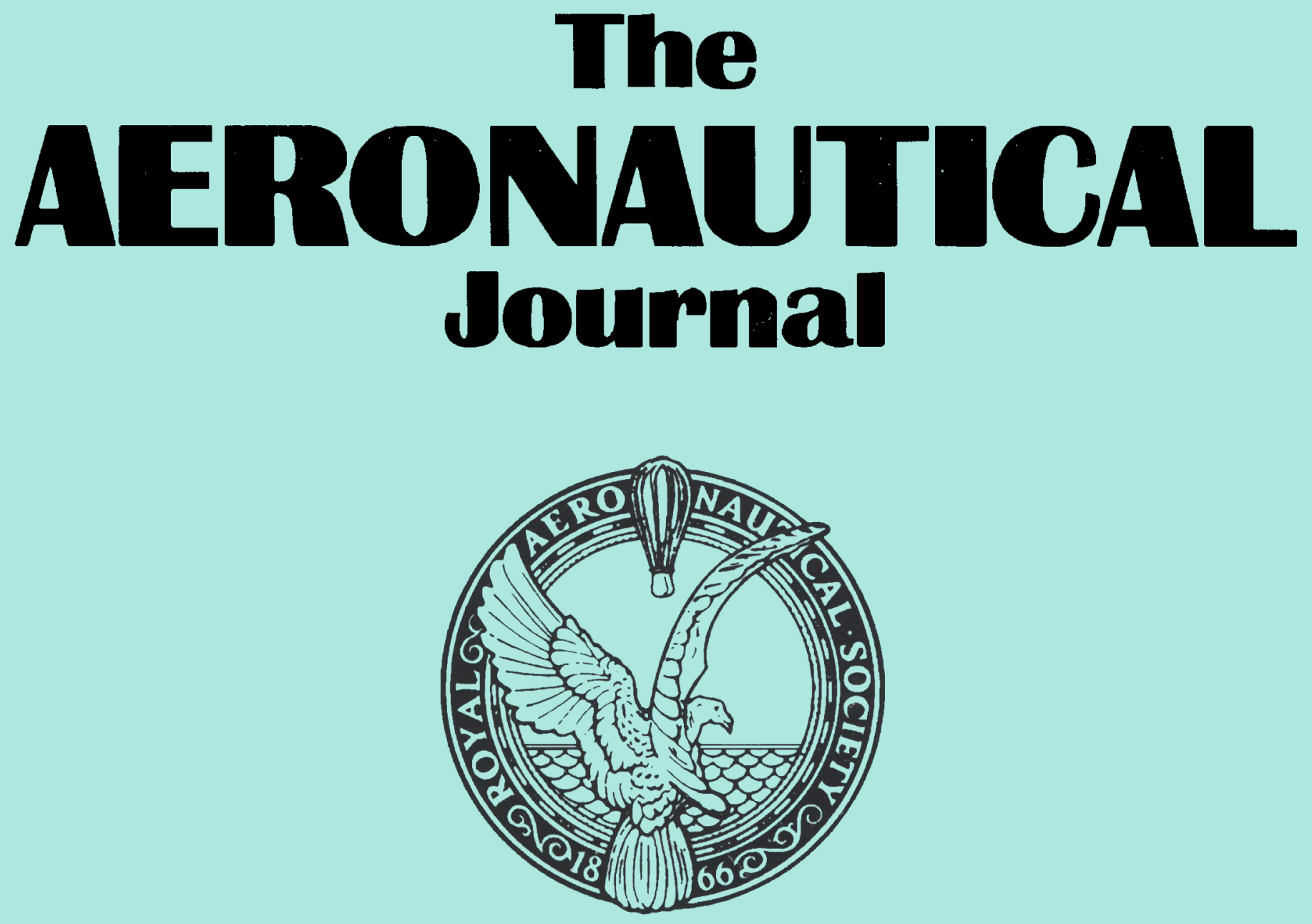

Contents

E.F. Toro and A. Chakraborty

The development of a Riemann solver for the steady supersonic Euler equations

D. Ambrosi, L. Gasparini and L. Vigevano

Full Potential and Euler solutions for transonic unsteady flow

R.B. Green and R.A. McD. Galbraith

Phenomena observed during aerofoil ramp-down motions from the fully separated state

\title{
C.R. Mclnnes
}

Compact analytical solutions for a decaying, precessing circular orbit 


\section{THE AERONAUTICAL JOURNAL}

\section{Aims and scope}

The aims and scope of The Aeronautical Journal are intended to reflect the objectives of the Society as expressed in the Charter of Incorporation. Briefly these are to encourage and foster the advancement of all aspects of aeronautical and space science. Thus the topics of the Journal include all those which are covered by the various Sections and Groups of the Society such as fluid mechanics and aerodynamics, propulsion, structures and materials, rotorcraft, astronautics and guided flight, dynamics and control, aeromarine technology, aviation medicine, air transport, test flying, flight simulation, air law, management studies, history of aviation and humanpowered flight. Thus papers are solicited on all aspects of research, design and development, construction and operation of aircraft and space vehicles. Papers are also welcomed which review, comprehensively, the results of recent research developments in any of the above topics. 


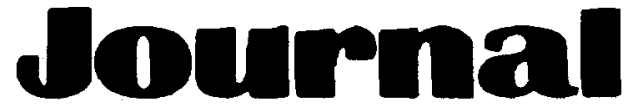

\title{
Editorial Advisory Board
}

\author{
Chairman: Dr E.W.E. Rogers
}

Editor

B.F. Baldwin, BSc, Associate Member RAeS

Deputy Editor

S.M. Penney, BEng, Grad RAeS

Editorial Assistants

C.S.C. Male, BSc(Eng), Grad RAeS

I.R. Sheppard, BEng, Grad RAeS

RAeS Director

R.J. Kennett, FIMgt, FInstD, FCIT, FRSA, AFAIAA, FRAeS

Published by

The Royal Aeronautical Society

4 Hamilton Place, London W1V OBQ.

Tel: $071-4993515$.

Fax: 071-4996230.

Advertisements

Chris Marot/Trevor Hornshaw

Marot \& Co

3 Albion Buildings

1 Back Hill, London EC1R 5EN

Tel: 071-278 3686

Fax: 071-837 2764

Reproduction of any of the papers

published in this journal is not

permitted without the written consent

of the Editor.

The content does not necessarily express the opinion of the Council.

Printed by

Manor Park Press,

Unit 7, Highfield Industrial Estate,

Edison Road, Hampden Park

Eastbourne.

Subscriptions: $£ 195$ a year, post free.

RAeS members: £22 a year.

Single copies, including back

numbers: $£ 24$.

Non-member subscriptions from

The Royal Aeronautical Society

Publications Subscriptions Department,

PO Box 139, Tonbridge,

Kent TN9 1EW, UK

Tel: 0732770823 .

Fax: 0732361708 .

ISSN: 0001-9240

The Royal Aeronautical Society is a registered charity.

PUBLISHED MONTHLY EXCEPT JUNE AND AUGUST
Aerodynamics

Professor P.W. Bearman, Department of Aeronautics, Imperial College

Aerospace medicine

Air Vice Marshal P. Howard, formerly RAF Institute of Aviation Medicine

Air traffic control and simulation

G.C. Howell, former Civil Aviation Authority chief scientist

Air transport

Professor R. Doganis, Department of Air Transport, Cranfield Institute of Technology

\section{Airworthiness}

R. Ashford, European Joint Aviation Authority

Avionics and systems

Professor D. McLean, Department of Aeronautics \& Astronautics, University of Southampton

P.A. Hearne, GEC-Marconi Avionics

Computational fluid mechanics and propulsion

Professor P. Stow, Rolls-Royce

Flight testing, flight operations and air transport

Captain W.D. Lowe, British Airways

Maintenance and airworthiness

G.B. Ratcliffe, RAeS Airworthiness and Maintenance Committee

Noise, aeroelasticity and fluid mechanics

Professor E.G. Broadbent, Department of Mathematics, Imperial College

Rotorcraft and structural dynamics

Professor D.E.H. Balmford, Westland Helicopters

\section{Space technology}

R. Gibson, Inmarsat

Structures and materials

T. Sharples, Military Aircraft Division, BAe Defence

Incorporating The Aeronautical Quarterly and the Journals

of The Institution of Aeronautical Engineers and

The Helicopter Association of Great Britain. 


\section{DIVISIONS AND BRANCHES OF \\ THE ROYAL AERONAUTICAL SOCIETY}

Australia Branches at: Adelaide, Brisbane, Canberra, Melbourne and Sydney.

President: Air Cdre W.J. BELTON, CEng, FRAeS. Hon. Secretary: R.D. BARKLA, CEng, MRAeS. PO Box 573, Mascot, NSW 2020.

New Zealand Branches at: Auckland, Blenheim, Canterbury, Hamilton, Otago, Palmerston North and Wellington.

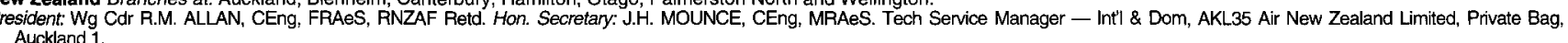

Pakistan President: S. AJAZ ALI, CEng, FRAeS. Hon. Secretary: Dr S.A. HASAN, CEng, FRAeS, 4th Floor, IEP Building, 177/2 Liaquat Barracks, Karachi 75530. Tel: $92-7780233$.

Southern Africa President: D.P. du PLOOY, CEng, MRAeS. Hon. Secretary. Ms LESLIE STOKOE, PO Box 9168, 1625 Eldeen, Republic of South Africa

Zimbabwe President: Wg Cdr E. CHINGOSHO, MRAeS. Hon. Secretary: A.S. MATIKITI, IEng, AMRAeS. Engineering Dept., Air Zimbabwe, PO Box 1931, Harare, Zimbabwe.

Bedford

President: R.J. WHYSALL, CEng, MRAeS

Chairman: G.W WOODS CEng, MRAES.

neecretary: C.I.P. MARTIN, AMRAeS. Hunting EngiTel: 0234841000 .

\section{Belfast}

President: T. STONE, FRAeS.

Chairman: Professor D. NIXON, FRAeS

Hon. Secretary: Dr S.T. MclLWAIN, Grad RAeS. Aerodynamics, Mechanical Engineering Group, Short Missile Systems, Montgomery Road, Belfast, Northern Ireland BT6 9HN. Tel: 0232465650

Birmingham, Wolverhampton and Cosford

President: S.L. HISCOX, MRAES.

Chairman: R JP HOWARD AMRAeS.

Hon. Secretary: A.R. BANBURY, CEng, Associate RAeS. 13 Grasmere Close, Tettenhall, Wolverhampton WV6 9DP. Tel: 0902745957

\section{Boscombe Down}

President: Air Cdre D.L. BYWATER, FRAeS, RAF.

Chairman: Dr D.W. WILLIAMS, CEng, FRAeS.

Hon. Secretary: Wg Cdr K. MACKENZIE, CEng, MRAeS ATG, Armament Division, A\&AEE Boscombe Down, Sal isbury SP4 0.J. Tel: 0980662975.

\section{Bristol}

President: S.J. SWADLING, CEng, FRAeS.

Chairman: D.C. GREENMAN, CEng, MRAeS

Hon. Secretary: J.G. MATTHEWS, Grad RAeS. Department B56, Technical Centre (C2), British Aerospace Airbus Ltd., Filton, Bristol BS99 7AR. Tel: 0272364006.

\section{Brough}

President: G. CHISNALL, CEng, MRAeS.

Chairman: S.J. BLEE, CEng, MRAeS.

Hon. Secretary: G.M. CAMMISH. Manufacturing and Structural Development, BAe (Military Aircraft) Brough, North Humberside HU15 1EQ. Tel: 0482664143.

\section{Cambridge}

President: Professor M. GASTER, FRS, CEng, FRAeS

Chairman: Wg Cdr P.N. GILBERT, CEng, MRAeS

Hon Secretary: D.N. WHITEHEAD, CEng, MRAeS. c/o Aircraft Design Office, Marshall Aerospace, The Airport, 373232/373541.

Cardiff

President: Gp Capt I.F. EASTON, CEng, FRAeS.

Chairman: J.A. STEELE, IEng, AMRAES

Hon. Secretary: Wg Cdr A.G. WILLENBRUCH, CEng, MRAeS. Engineering Wing, RAF St Athan, Barry CF6 OWA. Tel: 0446798798 Ex. 6053.

\section{Chester}

President: S.A.E. DYKE

Chairman: J.R.F. YOUNG, AMRAeS

Hon. Secretary: Dr J.E. ROBINSON. Padeswood Lodge,

Padeswood, Mold, Clwyd CH7 4JF. Tel: 0244546420

\section{Christchurch}

President: M.J. COBHAM, CBE, FRAeS

Chairman: K.W. NORRIS, FRAeS.

Hon. Secretary: M.G. PORTER, CEng, MRAeS. Flight Refueling, Brook Road, Wimborne, Dorset BH21 2BJ. Tel: 0202882121.

Coventry

President: J.B. ROBERTS CEng, FRAES.

Chairman: Dr B.D. HOBSON, CEng, MRAeS.

Hon. Secretary: R.I. RATCLIFF, IEng, Technician RAeS. 6 Erithway Rd, Green Lane, Coventry CV3 6JT. Tel: 0203 413779 .

Cranwell

President: Air Vice Marshal D. COUSINS, RAF.

Chairman: Wg Cdr M.F.J. NETHAWAY.

Hon. Secretary: Sqn Ldr J.E. MORTON, CEng, MRAeS. RAF College Cranwell, Sleaford, Lincs NG34 8HB. Derby

Derby

President: J.O. KEIR, FRAES.

on. Secretary: K. COBLEY, CEng, MRAeS. c/o Compres sor Technology, Rolls-Royce plc, PO Box 31, Derby DE24 8BJ. Tel: 0332249017.

\section{Gatwick}

President: M.J. WILLETT, FRAeS.

Chairman: A.J. HEATH, OBE, CEng, FRAeS.

Hon. Secretary: S.A. WITTS, MRAeS. Civil Aviation Authority, Transport Aeroplane Group, Aviation House, Gatwick RH6 OYA. Tel: 0293573285.

\section{Glasgow}

Chairman: F.J. MULLLEN

Murray 7, East Killbride G75 OLU. Tel: 0355263620 .
Miville

\section{Gloucester and Cheltenham}

President: D.G.M. DAVIES, OBE, CEng, FRAeS

Chairman: R.H. ASHFORTH, CEng, FRAeS

Hon. Secretary: R.F.J. McCARTHY, CEng, FRAeS. Dowty Aerospace, Anson Business Park, Cheltenham Road, Gloucester GL2 9QH. Tel: 0452711428 .

\section{Hattield}

President: F.W. VANN, CEng, FRAeS

Chairman: F.B. OGILVIE, AMRAeS.

Hon. Secretary: D.H. TIPPER, CEng, MRAeS. 4 Bishops Rise, Hatfield, Herts AL 10 9HB. Tel: 0707271025.

\section{Heathrow}

President: SIR COLIN MARSHALL, Hon Companion RAeS. Chairman: K.D. MITCHELL, Associate Member RAeS.

Hon. Secretary: R.A. KENNEDY, IEng, AMRAeS. British Airways (S.1) PO Box 10, Heathrow Airport, Hounslow, Middx TW6 2JA. Tel: 081-562 3366 .

\section{Isle of Wight}

President: R.L. WHEELER, CEng, FRAeS

Chairman: R.L. WHEELER, CEng, FRAeS.

Hon. Secretary: P.J. CHIVERS, CEng, MRAeS Westland Aerospace, Castle Street, East Cowes, Isle of Wight PO32 6RH. Tel: 0983294101 Ex. 2016.

\section{Loughborough}

President: Professor G.L. WILDE, OBE, CEng, FRAeS

Chairman: R.C.T. HARDING, Student RAeS.

Hon. Secretary: Professor S.J. STEVENS, CEng, MRAeS. Transport Technology Dept, Loughborough University of Technology, Leics. LE11 3TU. Tel: 0509223403. Luton

President: B. NEWTON

Chairman: P.J. SHARP, CEng, MRAeS.

Hon. Secretary: N.G. TOOMEY, IEng, MRAeS. c/o Monarch Aircraft Engineering Ltd (Planning Dept), Luton International Airport, Luton, Beds LU2 9LX Tel: Work-0582 398542. Home-0234 54185 .

Manchester

President: Professor J.B. SCOTT-WILSON, OBE, FEng, FRAeS.

Chairman: A A MCDICKEN, MRAeS,

Hon. Secretary: I.R. FORD, Student RAeS. PS50, Avro International Aerospace, Woodford, Stockport, Cheshire SK7 IQR. Tel: 061-957 4586 .

\section{Marhar}

President: Gp Capt. G.E. STIRRUP FRAeS

Chairman: Sqn Ldr R.B. ECKERSLEY, CEng, MRAeS

Hon. Secretary: Flt Sgt N.P. KING, AMRAeS. 617 Squadron RAF Marham, Kings Lynn, Norfolk PE33 9NP. Tel: 0760 337261 Ex. 6423.

\section{Medway}

President: P. A. HEARNE, FEng, Hon. FRAes.

Chairman: G.R. SLEIGHT, CEng, FRAeS,

Hon. Secretary: R.T. TWINE, MRAeS. c/o GEC-Marconi Avionics Ltd, Airport Works, Rochester, Kent ME1 2XX. Avionics Ltd, Airport Works,
Tel: 0634844400 Ex. 3534.

\section{Middle Wallop}

President: Col W.G. HARRISON.

Chairman: Lt Col T.W. PERKS, MRAeS

Secretary: Major P. MILBORN, MBE, IEng, AMRAeS. OC Training Company, School of Aeronautical Engineering, Army Air Corps Centre, Middle Wallop, Stockbridge, Hampshire SO20 8DY. Tel: 0264384305.

\section{Preston}

President: Professor D. GARDNER, FEng, FRAeS.

Chairman: M. MANSELL, CEng, FRAeS

Hon. Secretary: M. GILCHRIST, BEng, Grad RAeS Weapons Management (W14), British Aerospace Defence, Warton Aerodrome, Preston, Lancs PR4 1AX. Tel: 0772855274 .

\section{Prestwick}

President: P. BERRY, MRAeS.

Chairman: D.R. SHEPHERD, CEng, MRAeS

Hon. Secretary: A.R. FROST, AMRAeS. 21 Fullerton Road, Prestwick KA9 2BU. Tel: 0702541616.

\section{Sherborne}

President: Position vacant

Chairman: P. SAUNDERS, IEng, AMRAeS

Hon. Secretary: J.D. SIMPSON, Student RAeS, 27 Mill Close, East Coker, Yeovil BA22 9LF. Tel: 0935702149.
Southampton

President: Professor G.M. LILLEY, CEng, FRAeS.

Chairman: P.P. BOYLE, CEng, MRAeS.

Hon. Secretary: D.R.J. BAXTER, CEng, FRAeS. Department of Aeronautics and Astronautics, University of Southampton, Highfield, Southampton, Hants SO9 5NH. Tel: 0703 593882

Southend

President: BARONESS PLATT of WRITTLE, CBE, FEng, FRAES.

Chairman: D. ROBERTS, Technician RAeS.

Hon. Secretary: Euring M. NICOLINI, MRAeS. 29 Symons Avenue, Leigh-on-Sea, Essex SS9 5QD.Tel: 0702 521554.

Stevenage

President: D.A. LAYBOURN, FCA, FRAeS

Chairman: Dr J.M. YOUNG, FRAeS.

Hon. Secretary: D. MURPHY, CEng, MRAeS. British Aerospace Space Systems, Gunnels Wood Road, Stevenage, Herts SG1 2AS. Tel: 0438736737

\section{Swindon}

President: Professor M.G. FARLEY, CEng, FRAeS.

Chairman: Wg Cdr T. KIRBY.

Hon. Secretary: V.A. SIMON, 20 Hawkswood, Covingham Park, Swindon, Wilts. Tel: 0793525350

\section{Weybridge}

President: Professor I.A. YATES, CBE, FEng, FRAeS.

Chairman: Miss J.M. GULLAND CEng, MRAeS

Hon. Secretary: K.J. DAVIES, CEng, MRAeS. 65 Stringers Avenue, Jacobs Well, Guildford, Surrey GU4 7NN. Tel: 048331529

Yeovil

President: V.A.B. ROGERS, CBE, FEng, FRAeS

Chairman: R.I. CASE, FEng, FRAeS

Hon Secretary: F.N.R. BALLAM, CEng, MRAeS. Box 285 , Westland Helicopters, Yeovil, Somerset BA20 2YB. Tel: 0935703779

\section{OVERSEAS BRANCHES}

Cyprus

President: D.M PANTAZIS, FRAeS.

Chairman:D.M PANTAZIS FRAeS

Hon. Secretary: C. PERICLEOUS, MRAeS. 8 Georgiou Christodoulou, Acropolis, Nicosia, Cyprus. Tel: $357-2$ 310430.

\section{Hong Kong}

Chairman: R. FAIRFIELD, FRAeS

Hon. Secretary: M.J. POMFRET, MRAeS. MME Dept HK Polytechnic, Kowloon, Hong Kong. Tel: Office 37666647. Home: 3-7213732.

Malaysia

Chairman: Dato R. SINGH, IEng, FRAeS.

Hon. Secretary: Lt Col A. LATIF B. TAHA, AMRAeS, MRAF. Dept of the Air Force, Ministry of Defence, Jalan Padang Tembak, 50634 Kuala Lumpur, Malaysia. Tel: 03-2354358.

Munich

President: Professor Dipl.Ing G. MADELUNG, FEng, HonFRAeS.

Hon. Secretary: G PARKER, Student RAeS. NAMMA, Postfach 1303, 82003 Unterhaching, Germany. Tel: 89-666 80140 .

\section{Northern Germany}

Chairman: Sqn Ldr A.C. MAJOR, CEng, MRAeS, RAF. Hon. Secretary: Sqn Ldr A.C. MAJOR, CEng, MRAeS, RAF. Puma Maintenance Flt, RAF Laarburch, BFPO 43 , Germany. Tel: 02837895367

\section{Republic of Ireland}

President: R.W. O'SULLIVAN, CEng, FRAes.

Chairman: H. REILLY, MRAES.

Hon. Secretary; J.J. CONERNEY, Technician RAeS. Eng. Wing, Air Corps, Baldonnel, Co. Dublin. Tel: 592493. Ex. 6633 .

Toulouse

President: J. PIERSON, FRAeS.

Chairman: A.S. McCLYMONT, FRAeS.

Hon. Secretary: W.A.N. FORD, CEng, FRAeS. Chrestias, Avenue du Bouconne, 31530 Lévignac, France. Tel: (33) 61854258 . 